\title{
Noninvasive Mechanical Ventilation: Models to Assess Air and Particle Dispersion
}

\author{
David S.C. Hui
}

Keywords

Exhaled air $\bullet$ Dispersion $\bullet$ NIV $\bullet$ Influenza $\bullet$ SARS

\section{$2.1 \quad$ Introduction}

Respiratory failure is a major complication of viral infections such as severe acute respiratory syndrome (SARS) [1], avian influenza H5N1 infection [2], and the 2009 pandemic influenza (H1N1) infection [3]. The course may progress rapidly to acute respiratory distress syndrome (ARDS) and multi-organ failure, requiring intensive care. Noninvasive ventilation (NIV) may play a supportive role in patients with severe viral pneumonia and early ARDS/acute lung injury. It can act as a bridge to invasive mechanical ventilation, although it is contraindicated in critically ill patients with hemodynamic instability and multi-organ dysfunction syndrome [4]. Transmission of some of these viral infections can convert from droplets to airborne during respiratory therapy.

During the major outbreak of SARS, endotracheal intubation [5], oxygen therapy, and NIV were found to be risk factors for major nosocomial outbreaks affecting health care workers [6]. Possible aerosol transmission during a nosocomial outbreak of seasonal influenza was temporally related to the application of NIV in an index patient with hypercapnic respiratory failure due to acute exacerbation of chronic obstructive pulmonary disease (COPD). The patient was on a medical ward with an imbalanced indoor airflow [7]. As influenza virus may be contained in fine

D.S.C. Hui, MD

Department of Medicine and Therapeutics, The Chinese University of Hong Kong,

Prince of Wales Hospital, 30-32 Ngan Shing St., Shatin, N.T., Hong Kong

Stanley Ho Center for Emerging Infectious Diseases, The Chinese University of Hong Kong, Shatin, N.T., Hong Kong

e-mail: dschui@cuhk.edu.hk 
particles generated during tidal breathing [8], NIV may disperse potentially infected aerosols, especially when patients cough and sneeze frequently, contributing to nosocomial transmission of influenza. Pulmonary tuberculosis (TB) is well known to spread by the airborne route. A recent study showed that a small number of patients with pulmonary TB (28\%) produced culturable cough aerosols [9].

Thus, it is important to examine the exhaled air directions and dispersion distances during application of NIV to patients with respiratory failure via commonly used face masks. The data can improve our understanding of and knowledge about infection control. Such knowledge can facilitate the development of preventive measures to reduce the risk of nosocomial transmission during application of NIV to high-risk patients with respiratory infections.

\subsection{Methods}

As there is no reliable, safe marker that can be introduced into human lungs for experimental purposes, the laser smoke visualization method and the human patient simulator (HPS) model have been adopted as the method for studying exhaled air dispersion during application of various types of respiratory therapy in hospital medical wards, including the negative-pressure isolation room [10-13].

\subsubsection{NIV and Lung Model}

The HPS represents a $70-\mathrm{kg}$ adult man sitting on a $45^{\circ}$-inclined hospital bed (Fig. 2.1). The HPS contains a realistic airway and is programmed to remove oxygen and inject carbon dioxide into the system according to a preset respiratory exchange ratio and oxygen consumption. The lung compliance can also be changed to simulate different degrees of lung injury during chest infection. By varying the oxygen consumption $(200,300$, and $500 \mathrm{ml} / \mathrm{min})$ and lung compliance $(70,35$, and $10 \mathrm{ml} / \mathrm{cmH}_{2} \mathrm{O}$ ), these sets of values produce a range of tidal volumes, respiratory rates, and peak inspiratory flow similar to those of patients with minimal (essentially normal lung function), moderate, or severe lung injury, respectively. For example, lung compliance is set at $35 \mathrm{ml} / \mathrm{cm} \mathrm{H}_{2} \mathrm{O}$ and oxygen consumption at $300 \mathrm{ml} / \mathrm{min}$ to mimic mild lung injury. Tidal volume and respiratory rate are regulated so a respiratory exchange ratio of 0.8 is maintained during measurements. Typically, this is achieved with a tidal volume of $300 \mathrm{ml}$ and a respiratory rate of 25 breaths/min [10-13]. Lung compliance and airway resistance also responds in a realistic manner to relevant respiratory challenges. The HPS produces an airflow pattern that is close to the in vivo situation. It has been applied in previous studies to simulate human respiration [14-17].

Deliberate leakage from the exhalation ports of the Mirage mask (ResMed, Bella Vista, NSW, Australia) [10], ComfortFull 2, and Image 3 masks (Respironics, Murrysville, PA, USA) [11] firmly attached to a high-fidelity HPS (HPS 6.1; Medical Education Technologies, Sarasota, FL, USA) has been evaluated. NIV was 


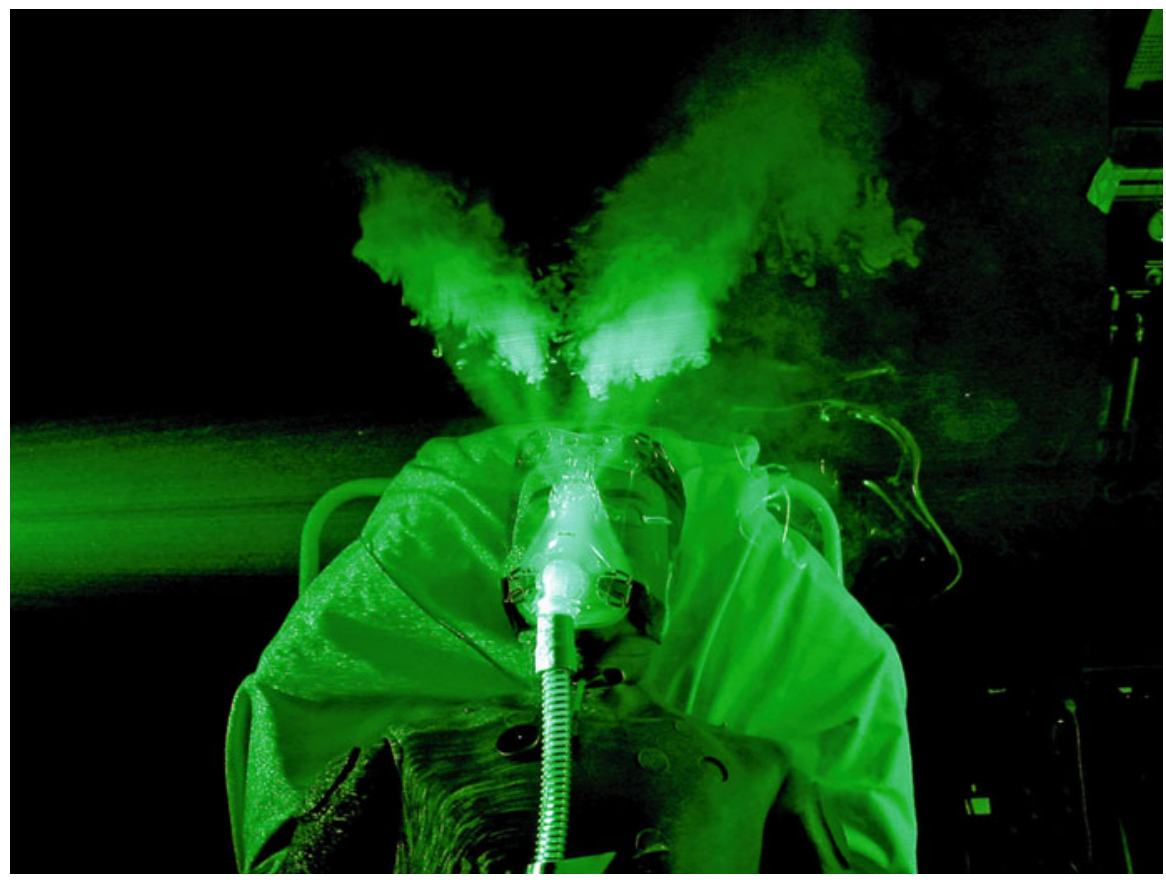

Fig. 2.1 Human patient simulator (HPS) lying at $45^{\circ}$ on a bed undergoing noninvasive ventilation via the ResMed Mirage face mask. A laser beam located on the right side of the bed lateral to the human patient simulator illuminates the exhaled air particles leaking from the exhalation ports of the face mask in the coronal plane. A camera was positioned along the sagittal plane at the end of the bed to capture lateral dispersion of exhaled air illuminated by the laser device. Positions of the camera and the laser device would be exchanged when the exhaled air dispersion from the face mask is examined along the sagittal plane

applied using a bilevel positive airway pressure device (VPAP III ST; ResMed) via each mask. The inspiratory positive airway pressure (IPAP) was initially set at 10 $\mathrm{cmH}_{2} \mathrm{O}$ and gradually increased to $18 \mathrm{cmH}_{2} \mathrm{O}$. The expiratory positive airway pressure (EPAP) was maintained at $4 \mathrm{cmH}_{2} \mathrm{O}$ throughout the study [10, 11].

\subsubsection{Flow Visualization}

Visualization of airflow around each NIV face mask was facilitated by marking the air with smoke particles produced by a M-6000 smoke generator (N19; DS Electronics, Sydney, Australia), as in our previous studies [10-13]. The oil-based smoke particles, measuring less than $1 \mu \mathrm{m}$ in diameter, are known to follow the airflow pattern precisely with negligible slip [18]. The smoke was introduced continuously to the right main bronchus of the HPS. It mixed with alveolar gas and then was exhaled through the airway. Sections through the leakage jet plume were then revealed by a thin, green laser light sheet (532 nm wavelength, continuous-wave 
mode) created by a diode-pumped solid-state laser (OEM UGH-800 mW; Lambda Pro Technologies, Shanghai, China) with custom cylindrical optics to generate a two-dimensional laser light sheet [10-13].

The light sheet was initially positioned in the median sagittal plane of the HPS and subsequently shifted to paramedian sagittal planes. This allowed us to investigate the regions directly above and lateral to the mask and the patient [10-13].

All leakage jet plume images revealed by the laser light sheet were captured by a high-definition video camera-Sony high-definition digital video camcorder (HDR-SR8E; Sony, Tokyo, Japan); ClearVid complementary metal oxide semiconductor sensor (Sony) with a Carl Zeiss Vario-Sonnar T* Lens (Carl Zeiss, Jena, Germany) —with optical resolution of $1,440 \times 1,080$ pixels per video frame. The normalized smoke concentration in the plume was estimated from the light intensity scattered by the smoke particles [10-13].

\subsubsection{Image Analysis}

The normalized smoke concentration in the mask leakage air was estimated from the light scattered by the particles. The analysis was based on scattered light intensity being proportional to the particle concentration under the special conditions of constant-intensity laser light sheet illumination and monodispersion of small (submicron) particles [18]. In short, the thin laser light sheet of near-constant intensity illuminated the smoke particle markers in the mask airflow leakage. Smoke particles scattered laser light perpendicular to the light sheet. The pictures were then collected and integrated by the video camera element and lens [10-13].

\subsubsection{Image Capture and Frame Extraction}

A motion video of at least 20 breathing cycles for each NIV setting was captured and individual frames extracted as gray-scale bitmaps for intensity analysis. Frames were extracted at time points starting from the beginning of each inspiration to generate an ensemble average for the corresponding instant of the respiratory cycle [10-13]. The time at which the normalized concentration contours spread over the widest region from the NIV mask was chosen for the ensemble average to estimate the greatest dispersion distance. This was found to be approximately at the mid-respiratory cycle $[10,11]$.

\subsubsection{Intensity Averaging and Concentration Normalization}

All gray-scale frames were read into a program specifically developed for these studies [10-13] (MathCad 8.0; MathSoft, Cambridge, MA, USA) [19] along with the background intensity images obtained with the laser switched off. The background intensity image was subtracted from each frame, pixel by pixel, to remove any stray background light. The pixel intensity values were averaged over all frames 
to determine the average intensity. The resulting image was the total intensity of light scattered perpendicular to the light sheet by the smoke particles. It was directly proportional to the smoke concentration under the conditions mentioned above. The image was normalized against the highest intensity found within the leakage jet plume to generate normalized particle concentration contours [10-13].

As the smoke particles marked air that originated from the HPS's airways before leaking from the mask, the concentration contours effectively represent the probability of encountering air around the patient that has come from within the mask and the patient's respiratory system. The normalized concentration contours are made up of data collected from at least 20 breaths. A contour value of 1 indicates a region that consists entirely of air exhaled by the patient, where there is a high chance of exposure to the exhaled air, such as at the mask exhaust vents. A value near 0 indicates no measurable air leakage in the region and a small chance of exposure to the exhaled air [10-13].

\subsection{Results}

The results are presented with reference to the median sagittal plane.

\subsubsection{Noninvasive Positive-Pressure Ventilation Applied via the ResMed Mirage Mask}

With the ResMed Mirage mask, a jet plume of air escaped through the exhaust holes to a distance of approximately $0.25 \mathrm{~m}$ radially during application of IPAP 10 $\mathrm{cmH}_{2} \mathrm{O}$, with some leakage from the nasal bridge. The leakage jet probability was highest about $60-80 \mathrm{~mm}$ lateral to the sagittal plane of the HPS. Without nasal bridge leakage, the plume jet from the exhaust holes increased to a $0.40 \mathrm{~m}$ radius circle, and exposure probability was highest about $0.28 \mathrm{~m}$ above the patient. When IPAP was increased to $18 \mathrm{cmH}_{2} \mathrm{O}$, the vertical plume extended to about $0.5 \mathrm{~m}$ above the patient and the mask, with some horizontal spread along the ward roof [10].

\subsubsection{Noninvasive Positive-Pressure Ventilation Applied via the ComfortFull 2 Mask}

With the ComfortFull 2 mask, a vertical, cone-shaped plume leaked out from the mask exhalation diffuser and propagated well above and almost perpendicular to the patient at an IPAP and an EPAP of 10 and $4 \mathrm{cmH}_{2} \mathrm{O}$, respectively. The maximum dispersion distance of smoke particles-defined as the boundary with a region encountering $<5 \%$ normalized concentration of exhaled air (light blue contour smoke concentration scale) - was $0.65 \mathrm{~m}$, whereas that of a high concentration (containing $>75 \%$ normalized concentration of exhaled air, red zone, and above) was $0.36 \mathrm{~m}$. There was no significant room contamination by exhaled air (as reflected by the blue background in the isolation room) other than the exhalation jet plume [11]. 


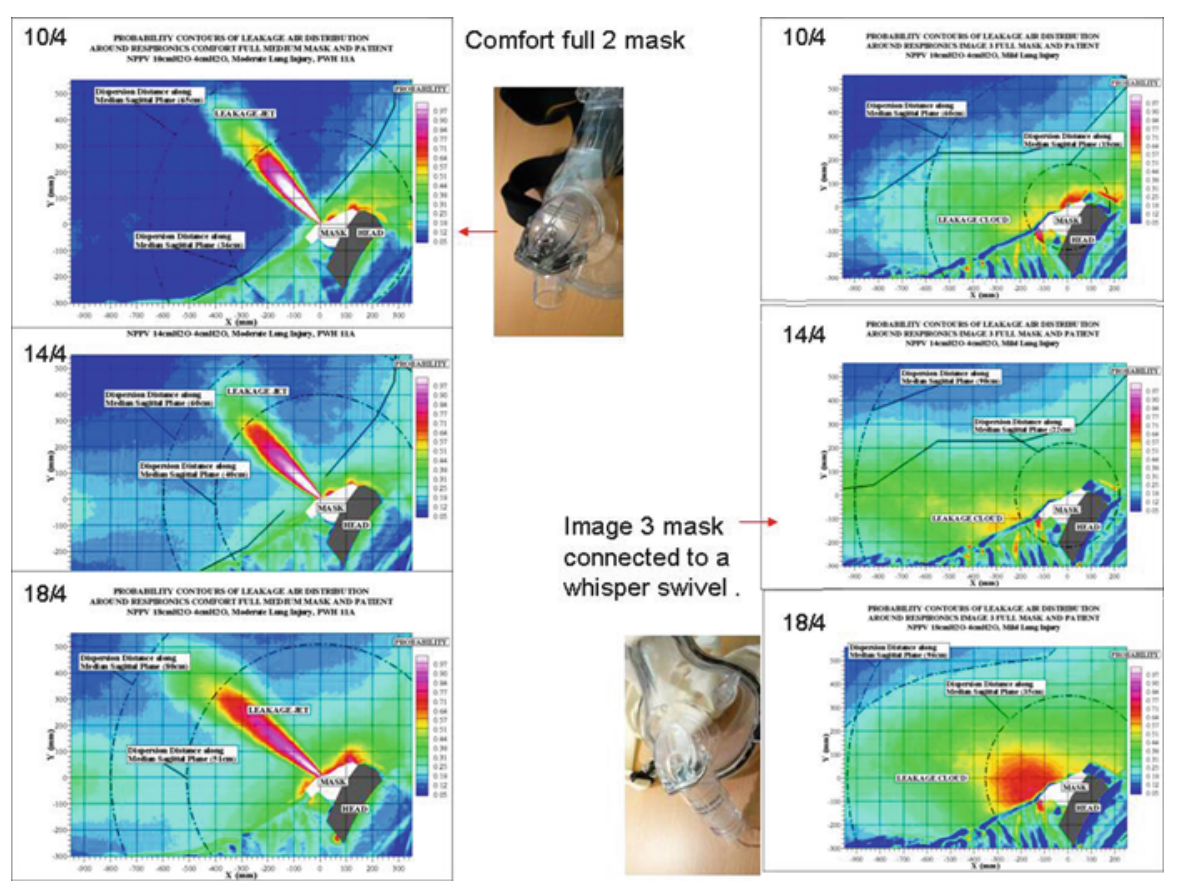

Fig. 2.2 Exhaled air dispersion along the median sagittal plane when the recumbent HPS was wearing the Comfortfull 2 mask (images on the left) or the Image 3 mask connected to the whisper swivel (images on the right) when the inspiratory positive airway pressure was increased from 10 to 14 and then $18 \mathrm{cmH}_{2} \mathrm{O}$ while the expiratory positive airway pressure was fixed at $4 \mathrm{cmH}_{2} \mathrm{O}$ [11]

When the IPAP was increased from 10 to $14 \mathrm{cmH}_{2} \mathrm{O}$, the maximum exhaled dispersion distance of low-concentration exhaled air was similar at $0.65 \mathrm{~m}$, but that of high-concentration exhaled air increased to $0.40 \mathrm{~m}$, with contamination of the isolation room. Also, there was some exhaled air concentration outside the exhalation jet plume. When IPAP was increased to $18 \mathrm{cmH}_{2} \mathrm{O}$, the dispersion distance of lowconcentration exhaled air was $0.85 \mathrm{~m}$, whereas that of high-concentration exhaled air increased to $0.51 \mathrm{~m}$ along the median sagittal plane. More background contamination of the isolation room by smoke particles was noted at higher IPAPs owing to interaction between the downstream ceiling-mounted ventilation vent and the upstream exhaled air from the HPS (images at left in Fig. 2.2) [11].

\subsubsection{Noninvasive Positive-Pressure Ventilation Applied via the Image 3 Mask Connected to the Whisper Swivel}

The Image 3 mask required an additional exhalation device (whisper swivel) to prevent carbon dioxide rebreathing. The exhaled air leakage was much more diffuse than that with the ComfortFull 2 mask because of the downstream leakage of 
exhaled air through the whisper swivel exhalation port. At an IPAP of $10 \mathrm{cmH}_{2} \mathrm{O}$, the maximum dispersion distance of a low concentration in exhaled air (light blue zone on the smoke concentration scale) was $0.95 \mathrm{~m}$ toward the end of the bed, whereas that of a medium concentration (containing $>50 \%$ of the normalized concentration of exhaled air, green zone, and above) was about $0.6 \mathrm{~m}$ along the median sagittal plane. As the IPAP was increased from 10 to $14 \mathrm{cmH}_{2} \mathrm{O}$, the exhaled air with a medium concentration increased to $0.95 \mathrm{~m}$ toward the end of the bed along the median sagittal plane of the HPS [11].

When the IPAP was increased to $18 \mathrm{cmH}_{2} \mathrm{O}$, the exhaled air with a low concentration dispersed diffusely to fill up most of the isolation room (i.e., beyond $0.95 \mathrm{~m}$, as captured by the camera), whereas that with a medium concentration, occupying wider air space, was noted to spread $0.8 \mathrm{~m}$ toward the end of the bed, with accumulation of a high concentration of exhaled air (red zone on scale) within $0.34 \mathrm{~m}$ from the center of the mask, along the median sagittal plane of the HPS (images on the right in Fig. 2.2) [11].

\subsection{Discussion}

There is no reliable, safe marker that can be introduced into human lungs for experimental purposes. Hence, the maximum distribution of exhaled air, marked by very fine smoke particles, from the HPS during application of NIV using three face masks was examined by the laser smoke visualization method on a high-fidelity HPS model. The studies showed that the maximum distances of exhaled air particle dispersion from patients undergoing NIV with the ResMed Ultra Mirage mask was $0.5 \mathrm{~m}$ along the exhalation port [10]. In contrast, the dispersion distances of a low, normalized concentration of exhaled air through the ComfortFull 2 mask exhalation diffuser increased from 0.65 to $0.85 \mathrm{~m}$ at a direction perpendicular to the head of the HPS along the sagittal plane when IPAP was increased from 10 to 18 $\mathrm{cmH}_{2} \mathrm{O}$. There was also more background contamination of the isolation room at the higher IPAP [11]. Even when a low IPAP of $10 \mathrm{cmH}_{2} \mathrm{O}$ was applied to the HPS via the Image 3 mask connected to the whisper swivel exhalation port, the exhaled air leaked far more diffusely than from the ComfortFull 2 mask, dispersing a low normalized concentration of $0.95 \mathrm{~m}$ along the median sagittal plane of the HPS. The higher IPAP resulted in wider spread of a higher normalized concentration of smoke around the HPS in the isolation room with negative pressure [11].

Simonds et al. [20] applied the laser visualization method to assess droplet dispersion during application of NIV in humans with an optical particle sizer (Aerotrak 8220; TSI Instruments, High Wycombe, UK) and showed NIV as a droplet- (not aerosol-) generating procedure, producing droplets measuring $>10 \mu \mathrm{m}$. Most of them fell onto local surfaces within $1 \mathrm{~m}$ of the patient.

Noninvasive ventilation is an effective treatment for patients with respiratory failure due to COPD, acute cardiogenic pulmonary edema, or pneumonia in immunocompromised patients. However, evidence supporting its use in patients with pneumonia is limited. NIV was applied to patients with severe pneumonia caused by 
a 2009 pandemic influenza (H1N1) infection with a success rate of about $41 \%$. Although there were no reported nosocomial infections [21], there is a potential risk of applying NIV to patients hospitalized with viral pneumonia on a crowded medical ward with inadequate air changes [7]. In this regard, deliberate leakage via the exhalation ports may generate droplet nuclei and disperse infective aerosols through evaporation of water content of respiratory droplets, resulting in a superspreading event. Nonetheless, NIV was applied using a single circuit to treat patients effectively with respiratory failure due to SARS in hospitals with good infection control measures (including installation of powerful exhaust fans to improve the room air change rate and good protective personal equipment at a level against airborne infection). There were no nosocomial infections among the health care workers involved [22, 23]. In contrast, a case-control study involving patients in 124 medical wards of 26 hospitals in Guangzhou and Hong Kong identified the need for oxygen therapy and use of NIV as independent risk factors for superspread of nosocomial SARS outbreaks [6]. Similarly, a systematic review has shown a strong association between ventilation, air movement in buildings, and airborne transmission of infectious diseases such as measles, tuberculosis, chickenpox, influenza, smallpox, and SARS [24].

These studies of infection with the HPS model [10,11] and in humans [20] have important clinical implications for preventing future nosocomial outbreaks of SARS and other highly infectious conditions such as pandemic influenza when NIV is provided. NIV should be applied in patients with severe community acquired pneumonia only if there is adequate protection for health care workers because of the potential risk of transmission via deliberate or accidental mask interface leakage and flow compensation causing dispersion of a contaminated aerosol [10, 11]. Pressure necrosis may develop in the skin around the nasal bridge if the NIV mask is applied tightly for a prolonged period of time. Many patients loosen the mask strap to relieve discomfort. Air leakage from the nasal bridge is definitely a potential means of transmitting viral infections. Fitting a mask carefully is important for successful, safe application of NIV. Addition of a viral/bacterial filter to the breathing system of NIV, between the mask and the exhalation port, or using a dual-circuit NIV via full face mask or helmet without heated humidification may reduce the risk of nosocomial transmission of a viral infection $[11,25]$.

In view of the observation that higher ventilator pressures result in wider dispersion of exhaled air and more air leakage [10,11], it is advisable to start NIV with a low IPAP $\left(8-10 \mathrm{cmH}_{2} \mathrm{O}\right)$ and increase it gradually as necessary. The whisper swivel is an efficient exhalation device to prevent carbon dioxide rebreathing, but it would not be advisable to use such an exhalation port in patients with febrile respiratory illness of unknown etiology. This is especially true in the setting of an influenza pandemic with the high potential of human-to-human transmission for fear of causing a major outbreak of nosocomial infections. It is also important to avoid the use of high IPAP, which could lead to wider distribution of exhaled air and substantial room contamination [11].

There are some limitations regarding the use of smoke particles as markers for exhaled air. The inertia and weight of large droplets in an air-droplet two-phase flow would certainly cause them to have less horizontal dispersion than occurs with the continuous air carrier phase during which the particles travel with increased inertia and drag. However, evaporation of the water content of some respiratory droplets 
during coughing or sneezing when exposed to NIV may produce droplet nuclei suspended in air, whereas the large droplets fall to the ground in a trajectory pathway [10-13]. As smoke particles mark the continuous air phase, the data contours described refer to exhaled air. The results would therefore represent the "upper bound" estimates for dispersion of the droplets-which would be expected to follow a shorter trajectory than an air jet due to gravitational effects-but not fully reflect the risk of large-droplet transmission [10-13].

In summary, the laser visualization technique using smoke particles as a marker in the HPS model is a feasible means of assessing exhaled air dispersion during application of NIV and other modes of respiratory therapy [10-13]. Substantial exposure to exhaled air occurs within $1 \mathrm{~m}$ of patients undergoing NIV in an isolation room with negative pressure via the ComfortFull 2 mask and the Image 3 mask connected to the whisper swivel exhalation port. It must be noted that there is far more extensive leakage and room contamination with the Image 3 mask, especially at higher IPAPs [11].

Health care workers should take adequate precautions for infection control. They especially must pay attention to environmental air changes when providing NIV support to patients with severe pneumonia of unknown etiology complicated by respiratory failure.

\section{Key Major Recommendations}

- The laser visualization technique using smoke particles as markers in the HPS model is a feasible means of assessing exhaled air dispersion during application of NIV and other modes of respiratory therapy.

- Substantial exposure to exhaled air occurs within $1 \mathrm{~m}$ of patients undergoing NIV even in an isolation room with negative pressure.

- During application of NIV, it is advisable to choose face masks with predictable exhaled air directions and distances through the exhalation port without addition of the whisper swivel device.

- It is important to avoid using high inspiratory pressures and any face mask that requires connection to the whisper swivel exhalation port as they would lead to wider distribution of exhaled air and substantial room contamination.

Acknowledgment I thank the Research Fund for the Control of Infectious Diseases \#06060202 (Food \& Health Bureau, HKSAR) for supporting my studies financially.

\section{References}

1. Lee N, Hui DS, Wu A, et al. A major outbreak of severe acute respiratory syndrome in Hong Kong. N Engl J Med. 2003;348:1986-94.

2. Hui DS. Review of clinical symptoms and spectrum in humans with influenza A/H5N1 infection. Respirology. 2008;13 Suppl 1:S10-3.

3. Hui DS, Lee N, Chan PK. Clinical management of pandemic (H1N1) infection. Chest. 2010;137:916-25. 
4. Hui DS. Influenza A/H5N1 infection: other treatment options and issues. Respirology. 2008;13 Suppl 1:S22-6.

5. Fowler RA, Guest CB, Lapinsky SE, et al. Transmission of severe acute respiratory syndrome during intubation and mechanical ventilation. Am J Respir Crit Care Med. 2004;169:1198-202.

6. Yu IT, Xie ZH, Tsoi KK, et al. Why did outbreaks of severe acute respiratory syndrome occur in some hospital wards but not in others? Clin Infect Dis. 2007;44:1017-25.

7. Wong BC, Lee N, Li Y, Chan PK, et al. Possible role of aerosol transmission in a hospital outbreak of influenza. Clin Infect Dis. 2010;51:1176-83.

8. Fabian P, McDevitt JJ, DeHaan WH, et al. Influenza virus in human exhaled breath: an observational study. PLoS One. 2008;3(7):e2691.

9. Fennelly KP, Jones-López EC, Ayakaka I, et al. Variability of infectious aerosols produced during coughing by patients with pulmonary tuberculosis. Am J Respir Crit Care Med. 2012;186:450-7.

10. Hui DS, Hall SD, Chan MT, et al. Non-invasive positive pressure ventilation: an experimental model to assess air and particle dispersion. Chest. 2006;130:730-40.

11. Hui DS, Chow BK, Hall SD, et al. Exhaled air dispersion distances during application of noninvasive ventilation via different Respironics face masks. Chest. 2009;136:998-1005.

12. Hui DS, Hall SD, Chan MTV, et al. Exhaled air dispersion during oxygen delivery via a simple oxygen mask. Chest. 2007;132:540-6.

13. Hui DS, Chow BK, Hall SD, et al. Exhaled air and aerosolized droplet dispersion during application of a jet nebulizer. Chest. 2009;135:648-54.

14. Kuhlen R, Max M, Dembinski R, et al. Breathing pattern and workload during automatic tube compensation, pressure support and T-piece trials in weaning patients. Eur J Anaesthesiol. 2003;20:10-6.

15. Good ML. Patient simulation for training basic and advanced clinical skills. Med Educ. 2003;37 suppl 1:14-21.

16. Goodwin JA, van Meurs WL, Sa Couto CD, et al. A model for educational simulation of infant cardiovascular physiology. Anesth Analg. 2004;99:1655-64.

17. Lampotang S, Lizdas DE, Gravenstein N, Robicsek S. An audible indication of exhalation increases delivered tidal volume during bag valve mask ventilation of a patient simulator. Anesth Analg. 2006;102:168-71.

18. Soo SL. Fluid dynamics of multiphase systems. Toronto: Blaisdell Publishing Company; 1967.

19. MathSoft Inc. Mathcad 8.0 for windows, users guide. Cambridge, MA: MathSoft Inc; 2000.

20. Simonds AK, Hanak A, Chatwin M, et al. Evaluation of droplet dispersion during non-invasive ventilation, oxygen therapy, nebuliser treatment and chest physiotherapy in clinical practice: implications for management of pandemic influenza and other airborne infections. Health Technol Assess. 2010;14:131-72.

21. Masclans JR, Pérez M, Almirall J, et al. Early non-invasive ventilation treatment for severe influenza pneumonia. Clin Microbiol Infect. 2013;19(3):249-56. doi:10.1111/j. 1469-0691.2012.03797.x.

22. Cheung TM, Yam LY, So LK, et al. Effectiveness of noninvasive positive pressure ventilation in the treatment of acute respiratory failure in severe acute respiratory syndrome. Chest. 2004;126:845-50.

23. Han F, Jiang YY, Zheng JH, et al. Noninvasive positive pressure ventilation treatment for acute respiratory failure in SARS. Sleep Breath. 2004;8:97-106.

24. Li Y, Leung GM, Tang JW, et al. Role of ventilation in airborne transmission of infectious agents in the built environment- a multidisciplinary systematic review. Indoor Air. 2007;17:2-18.

25. Conti G, Larrsson A, Nava S, et al. On the role of non-invasive ventilation (NIV) to treat patients during the H1N1 influenza pandemic. ERS and ESICM guideline Nov 2009. Available at http://www.dev.ersnet.org/uploads/Document/63/WEB_CHEMIN_5410_1258624143.pdf 